\title{
Otoacoustic emissions and auditory brainstem responses in the newborn
}

\author{
C R Kennedy, L Kimm, D Cafarelli Dees, P I P Evans, M Hunter, S Lenton, R D Thornton
}

\begin{abstract}
The auditory function of $\mathbf{3 7 0}$ infants, drawn from both low and high risk groups, was tested before postnatal discharge using three tests: standard auditory brain stem responses (ABR), automated analysis of $\mathbf{A B R}$, and automated analysis of evoked otoacoustic emissions (OAE). All infants failing any neonatal test had further audiological evaluation. Follow up information was also available on those who passed neonatal tests. Automated OAE testing of both ears was quickest (median 12.5 minutes) and least invasive (no scalp electrodes). Bilateral failure rates (and upper $95 \%$ confidence limits) with a stimulus 35-36 dB above normal hearing threshold level (nHL) were $3.0 \%(4.6)$ with automated OAE, 3.2\% (5.1) with ABR, and $2.7 \%(4 \cdot 4)$ with automated ABR. Automated OAE was the test most sensitive for subsequently confirmed hearing impairment. Sequential testing with automated OAE followed, in those failing this test, by automated ABR would have provided a screening test for substantial hearing impairment with a specificity greater than $99 \%$ in this population. Possible application as a universal screen is discussed.
\end{abstract}

Children with severe to profound congenital hearing impairment-that is, a hearing loss $>60 \mathrm{~dB}$ relative to normal hearing threshold level (nHL)-will not develop speech and language normally. This impairment and the secondary cognitive and behavioural consequences can be devastating. There is some evidence that early rehabilitation is effective in reducing this impairment. ${ }^{1}$ The aim of an infant hearing screening programme should be to detect severe to profound hearing losses and to reduce the age at which intervention is begun. ${ }^{2}$

The American Academy of Pediatrics published a 'position statement' in 1982 which recommended that screening of infants at high risk of hearing impairment should be completed by 3 months and habilitation begun by 6 months of age. ${ }^{3}$ Screening of high risk groups rather than all infants was recommended. Indeed universal screening of hearing has never been widely adopted in North America. Unfortunately less than half of all severe/ profound hearing losses occur in infants with the current high risk factors: a recent thorough application of distraction testing to a cohort of high risk infants (weighing $<2000 \mathrm{~g}$ at birth or admitted to a special care baby unit for $>24$ hours) in the Oxford region was reported to be ineffective because $71 \%$ of infants in that birth cohort who were registered as hearing impaired before the age of 3 years were not in the 'at risk' group. ${ }^{4}$ This presents a strong argument in favour of broadening the definition of 'high risk' or introducing universal screening.

In the UK universal screening is currently performed in the form of distraction testing at 8 months. Although explicit guidelines for parents, direct access to audiology services, and improved health visitor training can help, ${ }^{5}$ the sensitivity and specificity of this test remain unacceptably low in most districts. ${ }^{67}$ Throughout the European Community, more than half of bilateral hearing losses of more than 50 $\mathrm{dBnHL}$ in the better ear remain undetected by the age of 3 years. ${ }^{8}$ Even in that minority who are detected by distraction testing, the diagnostic process is rarely complete before the age of 1 year so that intervention is necessarily delayed.

Neonatal testing offers the opportunity for earlier diagnosis and intervention. Automated behavioural tests such as the auditory response cradle $^{9}$ have not been sufficiently sensitive or specific to succeed as screening tools. ${ }^{1011}$ The auditory brain stem response (ABR) is established as a sensitive test for auditory dysfunction in the neonatal period with specificity which can be high provided testing is deferred until term and/or recovery from acute illness. ${ }^{12}$ Unfortunately $\mathrm{ABR}$ testing requires too much time per test and too much specialised training in interpretation to be suitable for universal screening. Automated analysis of ABR may help overcome these drawbacks.

The discovery of otoacoustic emissions $(\mathrm{OAE})^{13}$ and their subsequent application to newborn infants ${ }^{14-16}$ has opened a possible new avenue to quick and reliable screening. We report here our experience of automated analysis of both ABR and evoked OAE in comparison both with standard ABR and with subsequent hearing assessment in high risk and low risk newborns. Possible application of these tests as part of a universal neonatal screening test is discussed.

\section{Patients and methods}

The infants studied were inpatients at Princess Anne Hospital, Southampton, which has an annual rate of 5500 deliveries representing $\mathbf{9 0 \%}$ of all deliveries in the Southampton health district. The Department of Neonatal Medicine incorporates a special care baby unit. This unit has six intensive care cots including the only two regionally funded cots in the Wessex region. 
Three objective methods of evaluating auditory function were evaluated in three groups of neonates. (1) Low risk group: full term babies at low risk of deafness. (2) Special care baby unit group: those, other than group 3 below, admitted to the unit for more than 48 hours. (3) Family history or craniofacial malformation group: babies at increased risk of hearing impairment because of craniofacial deformity or a family history of deafness.

Both ears of each baby were tested by the following methods:

(1) ABR: measurement of ABR produced by series of clicks at 36,45 , and $60 \mathrm{dBnHL}$ was made via three scalp electrodes, an averaging computer, and a printer. The presence and latency of the $A B R$ were determined by a trained observer.

(2) Automated ABR analysis: the ABR produced by a series of clicks at $35 \mathrm{dBnHL}$ was detected as in (1), compared with a stored template of an ABR, and the response designated as 'pass' or 'refer' by an automated analyser (marketed as Algotek or Algo-1).

(3) Automated OAE analysis: OAE after presentation of clicks at 16,26 , and $36 \mathrm{dBnHL}$ were detected by a microphone within the same probe used to present the clicks in the canal of the external ear. Programmable otoacoustic measurement system (POEMS) software ${ }^{11}$ was used with an averaging computer to determine whether an emission was detectable. Evoked OAE were considered present if a visual display showed a response which was both visible on the screen and fulfilled statistical criteria calculated by POEMS. (Criteria were: (i) $\mathrm{F}_{\mathrm{sp}}{ }^{*}$ of signal in each series of computer-averaged emissions $>2$ and (ii) correlation coefficient between two series of computer averaged emissions $>0.5$.) The automated OAE analysis by POEMS controls all the test parameters and will provide prompts for the tester to follow in order to complete the test procedure quickly, simply, and in a standardised way. Different testers can therefore be employed with minimal training.

Additional information on the clinical history of the infant and on middle ear function (immittance) was also recorded in a standardised database. Subsequent follow up on all tested babies included the results of distraction testing at 8 months, questionnaires to the families when the child was 18 months' old, and the results of subsequent assessment in any tested baby seen at the District Preschool Hearing Clinic or the Wessex Regional Audiology Clinic. In addition, all babies failing any neonatal test and a random sample of those who passed had the neonatal tests repeated at the age of 3 months. All babies failing at 3 months were referred for a full audiological evaluation at the Wessex Regional Audiology Clinic.

The aim of the testing procedures was to detect hearing impairment of a degree likely to interfere substantially with the development of speech and language. We defined such 'substantial' hearing impairment as moderate $(>40$

${ }^{*}$ The $F_{\mathrm{sp}}$ is the $\mathrm{F}$ statistic for sound pressure level and provides a measure similar to signal-to-noise ratio.
$\mathrm{dBnHL}$ ) or greater hearing loss in the better ear. Crude failure rates on neonatal tests were derived. The significance of differences between percentage failure rates (\%diff) in the three groups of babies on a particular neonatal test was examined by calculating the standard error of the difference between the percentage failure rates (SE \%diff). If \%diff -SE \%diff is greater than 2 , then $\mathrm{p}<0.05$ that the difference arose by chance.

The false positive rate (that is, test failure without subsequently confirmed substantial hearing impairment), expressed as a percentage, is equal to 100 minus the specificity, expressed as a percentage. The percentage of false positives (and upper $95 \%$ confidence limit) for substantial hearing impairment was calculated for each test by subtracting cases of subsequently confirmed substantial hearing impairment from the crude failure rates. Cases in whom follow up assessment of hearing by audiology services was incomplete were assumed not to be hearing impaired for the purpose of specificity calculation, tending to underestimate the specificity of the failed neonatal test.

\section{Results}

Three hundred and seventy babies were tested and follow up, including results of distraction testing, is available on the first 354 . Testing on the low risk babies $(n=86)$ and those with a family history or craniofacial malformation $(n=61)$ was carried out at a median (quartiles) postconceptional age of $39(38 \cdot 3-41)$ weeks. For babies in the special care baby unit group $(\mathrm{n}=223)$ median birth weight was $2130 \mathrm{~g}$ and median gestational age was $34(32 \cdot 5-37)$ weeks at birth and $37(36-39)$ weeks at testing.

Practicability as reflected in test duration was compared between neonatal tests. Automated OAE took a median (5th and 95th centiles) time of $7 \cdot 2(5 \cdot 8-12 \cdot 5)$ minutes. Electrode placement, required for $\mathrm{ABR}$ and automated $\mathrm{ABR}$ but not for automated OAE, took $12 \cdot 5(8 \cdot 4-21)$ minutes. The additional testing time was less for automated ABR (1.5 (0.4-9.8) minutes) than for ABR (7.5 (6.7-11.8) minutes). For the latter test only, additional time and skills were needed to interpret the printed ABR. Testing with ABR, automated ABR and automated OAE was completed in 719,699 , and 714 ears respectively with neither ear tested in two $(0 \cdot 5 \%), 10(2 \cdot 7 \%)$, and six $(1 \cdot 6 \%)$ babies respectively. Failure to test either ear was attributable to failure of the baby to settle in none, nine, and four babies respectively with equipment failure accounting for the remainder. The number of such failures was undoubtedly increased by the need to complete all three types of testing plus immittance measurement in every infant in a single session.

\section{FAILURE RATES}

Failure rates (upper $95 \%$ confidence limit) for detection of an OAE in either ear after presentation of a 26 or $36 \mathrm{dBnHL}$ stimulus were $4.6 \%(6.8)$ and $3.0 \%(4.6)$ of all 370 infants tested respectively. Equivalent figures for 


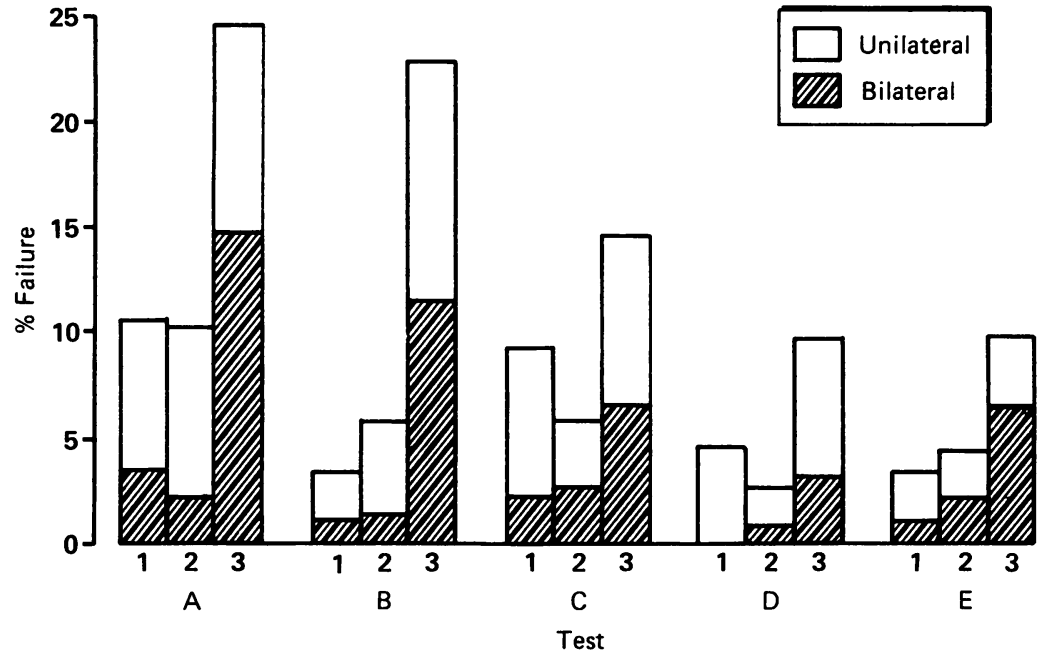

Figure 1 Percentage of infants failing a neonatal hearing test. Groups: $(1)=$ low risk $(n=86),(2)=$ special care baby unit infants (excluding group (3)) $(n=223)$, and $(3)=$ craniofacial malformation or family history of deafness $(n=61)$. Tests: $(A)=$ automated $O A E$ analysis with $26 \mathrm{dBnHL}$ stimulus; $(B)=$ automated $O A E$ analysis with $36 \mathrm{dBnHL}$ stimulus; $(C)=$ conventional $A B R$ with a $35 \mathrm{dBnHL}$ stimulus; $(D)=$ conventional $A B R$ with a $45 \mathrm{dBnHL}$ stimulus; and $(E)=$ automated $A B R$ analysis with a $35 \mathrm{dBnHL}$ stimulus.

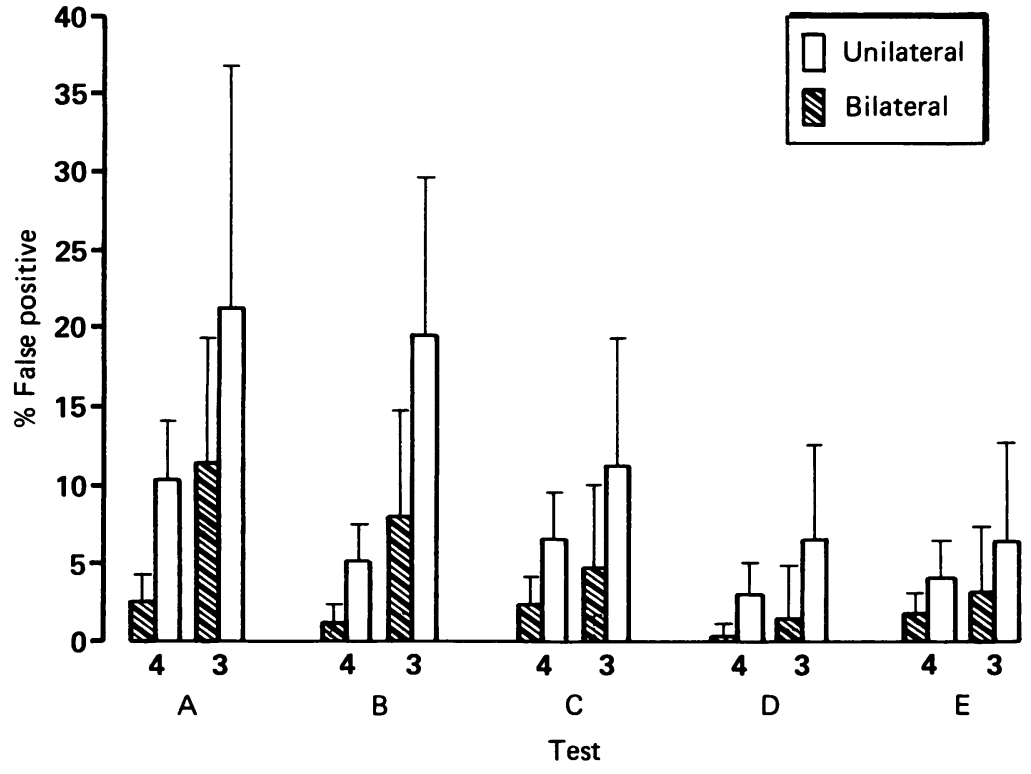

Figure 2 Percentage (with upper 95\% confidence limit) of infants failing a neonatal hearing test without subsequent confirmation of substantial hearing impairment (that is,

false positive rates). Groups: (4)=low risk plus special care baby unit infants (excluding group (3)) ( $n=309)$ and (3)=craniofacial malformation or family history of deafness $(n=61)$. Tests: $(A)=$ automated $O A E$ analysis with $26 \mathrm{dBnHL}$ stimulus; $(B)=$ automated $O A E$ analysis with $36 \mathrm{dBnHL}$ stimulus; $(C)=$ conventional $A B R$ with a $35 \mathrm{dBnHL}$ stimulus; $(D)=$ conventional $A B R$ with a $45 \mathrm{dBnHL}$ stimulus; and $(E)=$ automated $A B R$ analysis with a $35 \mathrm{dBnHL}$ stimulus.

percentage failure on $\mathrm{ABR}$ at 35 and $45 \mathrm{dBnHL}$ and for automated ABR screening at $35 \mathrm{dBnHL}$ were $3 \cdot 2 \%(5 \cdot 1), 1 \cdot 1 \%(2 \cdot 2)$, and $2 \cdot 7 \%(4 \cdot 4)$ respectively. Failure rates in the special care baby unit and low risk groups were very much alike (\%diff $<$ SE \%diff) while the babies who had a family history or craniofacial malformation had higher failure rates on all neonatal tests (fig 1). Because of the very similar failure rates in special care baby unit and low risk groups, these groups have been combined for the purpose of subsequent calculations of percentage failures and false positives (see discussion). Failure rates on automated OAE were significantly higher in the group with a family history or craniofacial malformation compared with the combined special care baby unit/low risk group (\%diff $>2$ $\times$ SE \%diff, $p<0.05$ with either a $26 \mathrm{~dB}$ or a 36 $\mathrm{dB}$ stimulus and with either unilateral or bilateral testing). ABR and automated ABR showed similar trends (\%diff $>$ SE \%diff and $<2 \times$ SE \%diff with all tests).

False positive rates as defined (see methods) are shown as percentages with upper $95 \%$ confidence limits for unilateral and bilateral testing in fig 2.

\section{SENSITIVITY}

Sensitivity of the neonatal tests for subsequently confirmed hearing loss was also examined. Thirteen cases of persistent hearing impairment were identified among the infants tested. Three came within our definition of substantial hearing impairment (see methods)(table). The remaining 10 had lesser degrees of impairment as detailed in the table and were included with infants who were not hearing impaired among the 'false positives' for those neonatal tests which they failed. Of these 10 , three infants who passed all neonatal tests were found to have persistent mild bilateral hearing impairment secondary to middle ear problems after failing distraction testing at 8 months (table). All three had normal immittance and tympanometry at the time of neonatal testing. The remaining seven infants with mild or moderate hearing impairments had all failed automated OAE in the neonatal period.

Questionnaires on hearing were sent to families of all 275 infants tested who have reached the age of 18 months and were completed by 210 $(76 \%)$ of these. Neither questionnaires nor distraction testing by health visitors at 8 months uncovered any other cases of hearing impairment nor did they come to district or regional audiology clinics by other routes.

PERFORMANCE OF TESTS IN COMBINATION Combinations of neonatal tests were considered. Automated OAE, being much the quickest and most convenient test, was the only possible contender as a universal screening tool and automated $\mathrm{ABR}$ was otherwise the test requiring the least time and skill and was highly specific (fig 2). Failure rates from a two stage screening procedure in which all automated OAE failures were screened with automated ABR were therefore derived for the same study population (fig 3). It is apparent that bilateral testing with automated OAE at either $26 \mathrm{dBnHL}$ or at 36 $\mathrm{dBnHL}$ followed by either unilateral or bilateral testing with automated ABR offers screening with an upper $95 \%$ confidence limit for 'false positive' failure of less than $1 \%$ (that is, a specificity with a lower $95 \%$ confidence limit of $>99 \%$ ).

\section{Discussion}

The suitability of a test for screening is dependent upon its specificity, sensitivity, and practicability. This study aimed to determine specificities of neonatal tests of auditory function for 
Results in all hearing impaired infants

\begin{tabular}{|c|c|c|c|c|c|c|c|c|}
\hline \multirow{2}{*}{$\begin{array}{l}\text { Patient } \\
\text { No }\end{array}$} & \multirow[t]{2}{*}{ Group } & \multicolumn{2}{|c|}{ Outcome hearing loss } & \multicolumn{4}{|c|}{ Neonatal test results (left/right) } & \multirow{2}{*}{$\begin{array}{l}\text { Distraction } \\
\text { test at } \\
8 \text { months }\end{array}$} \\
\hline & & $\begin{array}{l}\text { Severity } \\
\text { (left/right) }\end{array}$ & Type & $\begin{array}{l}\text { Automated } \\
O A E^{*}\end{array}$ & $A B R^{*}$ & $\begin{array}{l}\text { Automated } \\
A B R\end{array}$ & $\begin{array}{l}\text { Immittance } \\
\text { and tympano- } \\
\text { metry }\end{array}$ & \\
\hline \multicolumn{9}{|c|}{ Substantial hearing impairment } \\
\hline $\begin{array}{l}1 \\
2 \\
3\end{array}$ & $\begin{array}{l}\text { Craniofacial malformation } \\
\text { Family history } \\
\text { Craniofacial malformation }\end{array}$ & $\begin{array}{l}40-60 / 40-60 \\
90 / 90 \\
60-90 / 40-60\end{array}$ & $\begin{array}{l}\text { Bilateral atresia } \\
\text { Sensorineural } \\
\text { Sensorineural and } \\
\text { bilateral atresia }\end{array}$ & $\begin{array}{l}>36 />36 \\
>36 />36 \\
>36 / 36\end{array}$ & $\begin{array}{l}60 />60 \\
? />60 \\
60 />60\end{array}$ & $\begin{array}{l}\text { Fail/fail } \\
\text { Fail/fail } \\
\text { Fail/fail }\end{array}$ & $\begin{array}{l}\text { Obstructed } \\
\text { Normal } \\
\text { Obstructed }\end{array}$ & $\begin{array}{l}\text { Not tested } \\
\text { Not tested } \\
\text { Not tested }\end{array}$ \\
\hline \multicolumn{9}{|c|}{ Mild hearing impairment } \\
\hline $\begin{array}{r}4 \\
5 \\
6 \\
7 \\
8 \\
9 \\
10 \\
11 \\
12 \\
13\end{array}$ & $\begin{array}{l}\text { SCBU } \\
\text { Family history } \\
\text { Craniofacial malformation } \\
\text { Family history } \\
\text { SCBU } \\
\text { Craniofacial malformation } \\
\text { Craniofacial malformation } \\
\text { SCBU } \\
\text { Craniofacial malformation } \\
\text { SCBU }\end{array}$ & $\begin{array}{l}20-40 / 20-40 \\
20-40 / \text { none } \\
20-40 / 20-40 \\
20-40 / 20-40 \\
40-60 / 20-40 \\
20-40 / 20-40 \\
20-40 / 40-60 \\
20-40 / 20-40 \\
20-40 / 40-60 \\
20-40 / 20-40\end{array}$ & $\begin{array}{l}\text { Conductive } \\
\text { Conductive } \\
\text { Conductive } \\
\text { Conductive } \\
\text { Conductive } \\
\text { Conductive } \\
\text { Conductive } \\
\text { Conductive } \\
\text { Conductive } \\
\text { Conductive }\end{array}$ & $\begin{array}{l}\text { Pass } \\
36 / \text { pass } \\
26 / 26 \\
\text { Pass } \\
26 />36 \\
\text { Pass/26 } \\
26 / 36 \\
\text { Pass } \\
\text { Pass } / 26 \\
\text { Pass } / 26\end{array}$ & $\begin{array}{l}\text { Pass } \\
35 t / \text { pass } \\
35 t / 35 t \\
\text { Pass } \\
\text { ?/? } \\
\text { Pass/pass } \\
\text { 45/45 } \\
\text { Pass } \\
\text { Pass/pass } \\
45 / 60\end{array}$ & $\begin{array}{l}\text { Pass } \\
\text { Pass/pass } \\
\text { Pass/pass } \\
\text { Pass } \\
\text { Fail/pass } \\
\text { Pass/pass } \\
\text { Pass/fail } \\
\text { Pass } \\
\text { Pass/pass } \\
\text { Fail/fail }\end{array}$ & $\begin{array}{l}\text { Pass } \\
\text { Pass/pass } \\
\text { Pass/pass } \\
\text { Pass } \\
\text { Flat/flat } \\
\text { Pass/pass } \\
\text { Pass/pass } \\
\text { Pass } \\
\text { Flat/flat } \\
\text { Pass/flat }\end{array}$ & $\begin{array}{l}\text { Fail } \neq \\
\text { Not tested } \\
\text { Pass } \\
\text { Fail } \neq \\
\text { Not tested } \\
\text { Fail } \\
\text { Fail } \\
\text { Fail } \neq \\
\text { Fail } \\
\text { Fail }\end{array}$ \\
\hline
\end{tabular}

${ }^{*}$ Results given as minimum stimulus intensity in $\mathrm{dBnHL}$ at which ABR or OAE (POEMS) was recorded.

†ABR present but wave latencies prolonged.

$\Varangle$ All infants except these three were known by health visitors to be attending audiology clinics when tested at 8 months.

†All infants except these three
SCBU: special care baby unit.

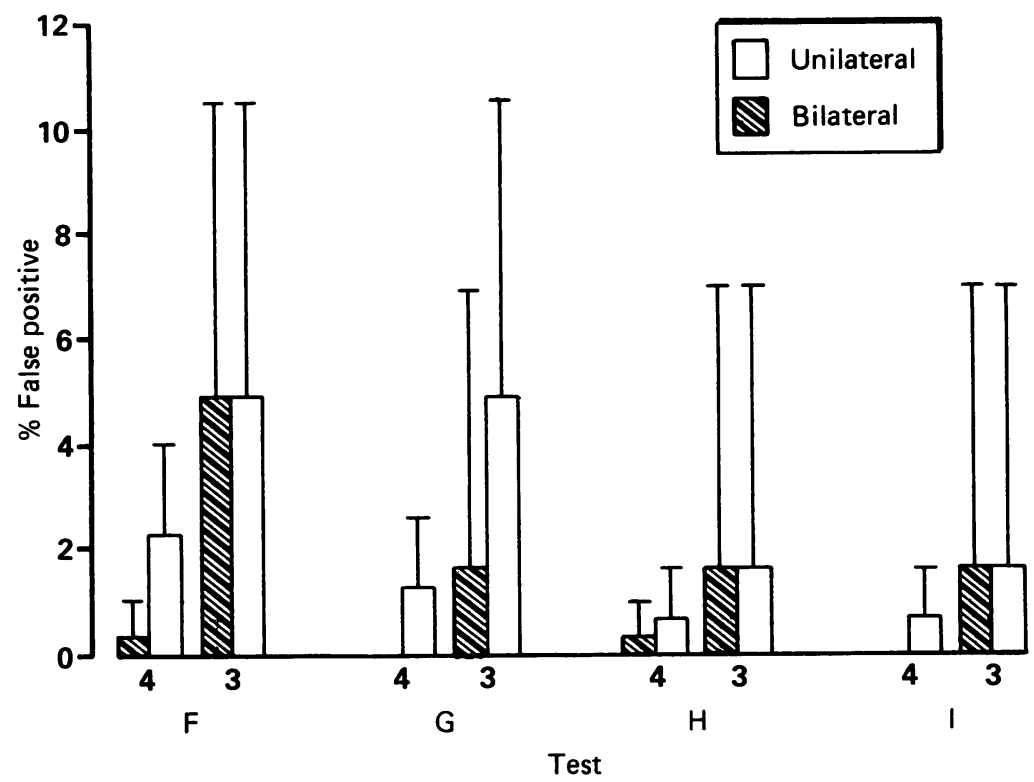

Figure 3 Percentage (with upper 95\% confidence limit) false positive rates on sequential combinations of neonatal tests. Groups: (4) low risk plus special care baby unit infants (excluding group (3)) $(n=309)$ and (3) craniofacial malformation or family history of deafness $(n=61)$. Tests: $(F)=$ automated $O A E$ analysis with $26 \mathrm{dBnHL}$ stimulus plus unilateral failure on automated $A B R ;(G)=$ as $(F)$ but with $36 \mathrm{dBnHL}$ stimulus for $O A E$;

$(H)=$ automated $O A E$ analysis with $26 \mathrm{dBnHL}$ stimulus plus bilateral failure on automated $A B R$; and $(I)=a s(H)$ but with $36 \mathrm{dBnHL}$ stimulus for $O A E$. In the figure unilateral and bilateral refer to failure on automated $O A E$ analysis.

severe hearing impairment but some observations about the other two parameters are also possible.

\section{SPECIFICITY}

Given that over three quarters of our study population were at increased risk of hearing impairment, the overall bilateral (unilateral) failure rates using automated OAE were low at approximately $6 \%(13 \%)$ and $3 \%(8 \cdot 1 \%)$ with a 26 and $36 \mathrm{dBnHL}$ stimulus respectively and these rates were reduced by more than half in both low risk and special care baby unit subgroups (fig 1).

These figures compare favourably with failure rates on a first and on both a first and a second 8 month distraction test of $16.3 \%$ and $6.8 \%$ respectively in a cohort of unselected children and of $18 \cdot 3 \%$ and $.3 \cdot 2 \%$ respectively in a population of babies from a special care baby unit. $^{46}$ An onward referral rate of $2 \cdot 6-6 \cdot 6 \%$ after failure on distraction testing over an entire health district was reported by Scanlon and Bamford. ${ }^{7}$ The failure-or test abandonedrate on a first test using the Linco-Bennett auditory response cradle was also higher at $7 \cdot 9 \%$ and $10.5 \%$ in two reports on both low risk and special care baby unit infants $9{ }^{11}$ and was $21 \%$ in a population from a special care baby unit. ${ }^{10}$

Others have reported that emissions can be recorded in $98-100 \%$ of normal infants. ${ }^{14} 16 \mathrm{~A}$ higher unilateral failure rate of $19-20 \%$ on OAE without automated analysis using a $41 \mathrm{dBnHL}$ stimulus has been reported in a special care baby unit population in which the ABR failure rate was similar to that in the present study. ${ }^{18}$ Differences between studies in the method of OAE analysis might account for the difference in OAE failure rate: our reliance upon explicit POEMS criteria (see methods) may have improved the specificity. It is also possible that Stevens et al tested a proportion of infants before they reached term before transfer to other units, although one would expect this to have been reflected in higher ABR failure rates: in our study almost all babies from the special care baby unit group were tested in the week before discharge to home.

As the aim of neonatal screening is to intervene early to minimise the effect of hearing impairment severe enough to interfere substantially with the development of speech and language, we elected to regard detection of lesser degrees of hearing impairment as 'false positives' in the calculation of specificity and sensitivity. This also avoided contamination of evaluation of the neonatal tests by the inclusion of mild/moderate conductive losses whose incidence rises rapidly in the secoufd six months of life and for which the benefits of intervention are debatable. ${ }^{19}$

The higher false positive rate in the group with a family history or craniofacial malformation (fig 2) was not associated with a higher rate of 
middle ear dysfunction on neonatal immittance measurement than the other two groups combined (10/51 compared with 60/260). It was, however, associated with a higher incidence of subsequently confirmed mild persistent conductive hearing loss (6/61 compared with 4/309) (table). This difference is sufficient to explain the higher false positive rate entirely and these two observations together suggest that neonatal automated OAE are a more sensitive predictor than neonatal immittance of subsequent conductive hearing loss.

Combination of groups of low risk and special care baby unit babies did not conceal a high false positive rate in the special care baby unit group (fig 1). Failure rates in the combined group tend to be marginally higher than for low risk group alone but the upper $95 \%$ confidence limit is lower for the combined group than for the low risk group alone because of the larger number of infants in the combined group. The false positive rate in the low risk population is the most influential parameter in determining the test performance for universal screening as this group will be approximately tenfold larger than the special care baby unit group. It seems unlikely that the failure rate in the low risk population was underestimated by adding the special care baby unit group to the low risk group.

In this study automated ABR, although more invasive (requiring application of scalp electrodes) and more time consuming than automated OAE, proved highly specific. This in itself is an important observation but, as with the observations on automated OAE (see below), further study is needed to establish that this technique has the sensitivity of conventional ABR analysis.

The use of a combination of neonatal tests enabled us to derive several screening pass/fail criteria (for example, fail if bilateral failure on automated OAE followed by unilateral failure on automated ABR analysis) which could be relied upon to fail less than $1 \%$ of babies irrespective of whether they were nursed in the special care baby unit or the postnatal wards provided there was no craniofacial malformation and no family history of deafness.

\section{SENSITIVITY}

All three cases of substantial hearing loss (see methods) that have so far come to light by any method were detected bilaterally on all the neonatal tests. Furthermore, there were indications in the study that automated OAE is also a sensitive test for lesser degrees of hearing impairment: seven of the 10 cases of mild to moderate hearing impairment (all middle ear in type) failed neonatally on automated OAE. Among these 10, three had an abnormal neonatal tympanogram, four failed neonatal $A B R$, and three passed all neonatal tests (table). In other words, had the aim been to predict subsequent mild to moderate hearing loss, automated OAE would have been the most sensitive neonatal test.

This finding is in agreement with that of Stevens et al that 27 out of 29 infants who failed $\mathrm{ABR}$ at $53 \mathrm{dBnHL}$ in one ear and $43 \mathrm{dBnHL}$ in the other on neonatal testing were predicted by failure to produce OAE after presentation of a $41 \mathrm{dBnHL}$ stimulus. ${ }^{18}$ In that study neither of the infants failing on $A B R$ and passing $O A E$ testing neonatally was reported to have confirmed severe hearing loss on the most recent test: one had ABR thresholds of $30 \mathrm{dBnHL}$ and the other 'still shows an elevated ABR threshold at seven months'.

It is, however, important to stress that, in contrast to the conclusions relating to specificity, the sensitivity of neonatal OAE (or neonatal automated $\mathrm{ABR}$ ) in detection of hearing impairment has not been reliably determined by the present study nor by any study yet published: because of the small number of severely hearing impaired infants expected in these study populations, a tenfold larger study to calculate sensitivities with the same narrow confidence limits as were derived for specificity is required. Furthermore, a long follow up period to allow any missed impairments to come to light is needed.

\section{PRACTICABILITY}

Automated OAE is certainly the most practicable of the neonatal tests used in this study as no scalp electrodes are required. The median testing time for automated OAE of 12 minutes would be substantially reduced in a screening protocol with less comprehensive testing (for example one or two instead of three stimulus intensities). Automated ABR, although a lengthier technique than automated $\mathrm{OAE}$, was quicker than standard ABR testing and also avoided the need for the training and time to evaluate $A B R$ tracings.

\section{POSITIVE PREDICTIVE VALUE}

The sensitivity and specificity of a test must be considered in relation to the prevalence of the condition in the population in order to determine the positive predictive value of failing the test: that is the percentage of cases failing the test who will subsequently prove to have an underlying hearing impairment. Several studies, reviewed by Sancho et al, ${ }^{2}$ report a prevalence of severe to profound deafness of $2-3 / 1000$ in infancy. Assuming that a neonatal screen of automated OAE followed by automated ABR is indeed $100 \%$ sensitive for this condition, one can deduce from the incidence of the condition and from our derivation of a lower $95 \%$ confidence limit of the specificity of such a screen of greater than $99 \%$ (that is, less than $1 \%$ false positives) that the positive predictive value of such a screen could approach $20-30 \%$.

\section{FUTURE STUDIES}

Having established the specificity of the neonatal tests, we wish to establish whether a neonatal screening protocol should either replace the 8 month distraction test or be used as an adjunct to it or not be used at all. To this end, we are currently undertaking a feasibility study to determine what staff and equipment would be required to implement effectively a screening 
protocol for all newborn babies before discharge from hospital. We plan to follow the feasibility study with a screening study of sufficient size to determine the sensitivity of the method within narrow confidence limits. Haggard correctly suggests that high false positive rates are the main obstacle to credible universal neonatal screening for hearing impairment. ${ }^{20}$ If we can confirm the high specificity reported here in such a screening study and demonstrate that such a screen is effective in reducing the age at which intervention for severe to profound deafness is begun, the case for widespread introduction of such a screen will be greatly strengthened.

We are grateful for the help and cooperation of Dr Penny Watt in arranging audiological follow up. This study has been generously supported by the Hearing Research Trust, the National Deaf Children's Society, the Trustee's Savings Bank, the Wessex Medical School Trust, the Wessex Regional Health Authority, the Michael Sobell Foundation, and the Susannah Peake Charitable Trust.

1 Markides A. Age of fitting of hearing aids and speech intelligibility. $\mathrm{Br} \mathcal{F}$ Audiol 1986;20:165-8.

2 Sancho J, Hughes E, Davis A, Haggard MP. Epidemiology of hearing impairments in children and its application to screening. In: McCormick B, ed. Paediatric audiology 0-5 years. London: Taylor and Francis, 1988:1-35.

3 American Academy of Pediatrics-position statement. Joint committee on infant hearing. Pediatrics 1982;70:496-7.

4 Johnson A, Ashurst $H$. Screening for sensorineural deafness by health visitors. Arch Dis Child 1990;65:841-5.
5 McCormick B. Hearing screening by health visitors-a critical appraisal of the distraction test. Health Visitor 1983;56:449-51

6 Brown J, Watson E, Alberman E. Screening infants for hearing loss. Arch Dis Child 1989;64:1488-95.

7 Scanlon PE, Bamford JM. Early identification of hearing loss: screening and surveillance methods. Arch Dis Child 1990;65:479-84.

8 Martin JAM, Bentzen O, Colley JRT, et al. Childhood deafness in the European community. Scand Audiol 1981; de:165-74.

9 Bhattacharya J, Bennett MJ, Tucker SM. Longterm followup of newborns tested with the auditory response cradle. up of newborns tested with the

10 McCormick B, Curnock DA, Spavins F. Auditory screening of special care neonates using the auditory response cradle. Arch Dis Child 1984;59:1168-72.

11 Hall DM, Garner J. Feasibility of screening all neonates for hearing loss. Arch Dis Child 1988;63:652-3.

12 Murray AD, Javel E, Watson CS. Prognostic validity of auditory brainstem evoked response screening in newborn infants. Am $\mathcal{F}$ Otolaryngol 1985;6:120-31.

$13 \mathrm{Kemp}$ DT. Stimulated acoustic emissions from within the hearing system. $\mathcal{F}$ Acoust Soc Am 1978;64:1386-91.

14 Elberling C, Parbo J, Johnsen NJ, Bagi P. Evoked acoustic emissions: clinical application. Acta Otolaryngol (Stockh) 1985;suppl 421:77-85

15 Stevens JC, Webb HD, Hutchinson J, Connell J, Smith ME, Buffin JT. Click evoked otoacoustic emissions compared with brainstem electrical response. Arch Dis Child 1989;64: with brain.

16 Bonfils P, Dumont A, Marie P, Francois $M$, Narcy P. Evoked otoacoustic emissions in newborn hearing screening. Laryngoscope 1990;100:186-9.

17 Cope Y, Lutman M. Oto-acoustic emissions. In: McCormick B, ed. Paediatric audiology 0-5 years. London: Taylor and Francis, 1988:221-45.

18 Stevens JC, Webb HD, Hutchinson J, Connell J, Smith MF, Buffin JT. Evaluation of click evoked otoacoustic emissions in the newborn. Br $\mathcal{F}$ Audiol 1990;24:293-300.

19 Stewart-Brown S, Haslum MN. Screening for hearing loss in childhood; a study of national practice. $B M \mathcal{F} 1987 ; 294$ : 1387-9.

20 Haggard MP. Hearing screening in children-state of the art(s). Arch Dis Child 1990;65:1193-5. 